\title{
EL MARJAL PEGO-OLIVA: EVOLUCIÓN TEMPORAL DE LA FLORA DE MACROALGAS
}

\author{
E. A. Cantora1 Uiza' ${ }^{1}$ y M. Aboal Sanjurjo ${ }^{2}$ \\ ${ }^{1}$ Laboratorio de Ficología, Departamento de Biología, Facultad de Ciencias, Universidad Nacional Autónoma de \\ México, México, D.F. E-mail: eacu@hp.fciencias.unam.mx. ${ }^{2}$ Laboratorio de Algología, Departamento de \\ Biología Vegetal (Botánica), Facultad de Biología, Universidad de Murcia, España. E-mail: maboal@um.es
}

\section{RESUMEN}

El Marjal de Pego-Oliva es una de las zonas húmedas más importantes del litoral mediterráneo español y sirve de refugio a comunidades vegetales $\mathbf{y}$ animales, muy amenazadas en otros lugares. Las actividades humanas en la zona se remontan a la edad del bronce y todavía quedan restos de los asentamientos romanos y musulmanes. En la actualidad se trata de una importante zona del Levante Español en el cultivo del arroz y de cítricos. Este humedal presenta un gradiente de salinidad desde aguas dulces a salobres que se manisfiesta en el cambio de las comunidades de algas $\mathbf{y}$ plantas. La diversidad de macro $\mathbf{y}$ microalgas en la zona es alta, a pesar de que nunca ha sido estudiada de forma intensiva, $\mathbf{y}$ se desarrollan especies de afinidad tropical. El grado de singularidad de este enclave se hace patente al compararlo con los restantes humedales litorales de la vertiente española. Se comparan los datos obtenidos en este estudio con datos previos, los más antiguos de los cuales proceden de hace 20 años, en un intento de mostrar la evolución de la flora y hacer una llamada de atención sobre el interés de la conservación de la zona, hoy ya declarada parque natural, pero sometida todavía a un gran número de presiones. El número total de especies reconocido en la actualidad está probablemente muy por debajo del real, debido a que el enorme número de ambientes diferentes existente en la zona, muchos de ellos temporales, hacen su muestreo bastante complicado $\mathbf{y}$ a que se carece por completo de datos de algunos de los grupos algales.

Palabras clave: Marjal Pego-Oliva, Mediterráneo, humedal, macroalgas, diversidad, riqueza específica, evolución temporal, conservación, España, ecología

\section{ABSTRACT}

The Marjal Pego-Oliva is one of the most important littoral wetlands of the Spanish Mediterranean slope and functions as a refuge for aquutic plant communities, which are very restricted in distribution, in a Spnnish or even European context. Human settlements have occupied this zone since Bronze Age and nowadays some Roman and Moorish ruins can still be observed. This wetland is located in the area of highest rainfall of all the Autonomous Community of Valencia. The special climatic characteristics of the area explain the presence of species with tropical affinities. Citrics and rice are the main crops of the region. A gradient of salinity can be observed in the wetlandfromfreshwater to saltwater influencing the plant and algal communities living there. The macroalgae diversity is high, although algal communities have never been studied in depth. Macroalgae data obtained in the last twenty years were compared in an attempt to understand changes experienced by the wetland and to improve control measures to protect the area. The wetland is suffering various impacts, despite it being designated as a protected area (i.e. Natural Park). Species richness has clearly been underestimated. This is in part due to the complexity of the system, with a great number of temporary habitats, and partly to the total absence of data available of some algae groups.

Keywords: Marjal Pego-Oliva, Mediterranean basin, wetlands, macroalgae, diversity, species richness, temporal dynamics, conservation, Spain, ecology 


\section{INTRODUCCIÓN}

En los últimos años se ha incrementado notablemente el interés por el estudio de los humedales, que muchas veces representan enclaves de elevada biodiversidad y de un alto valor e interés ecológico (Adam, 1990; Mitsch \& Gosselink, 1993). En el litoral mediterráneo español existen numerosas zonas húmedas, que no sólo son de vital importancia en las rutas migratorias de las aves, sino que en muchos casos son claves en el desarrollo agrícola o turístico de las áreas en las que se encuentran localizados (Mataeche, 1998). De entre ellos los marjales mediterráneos constituyen ambientes de protección prioritaria en la Unión Europea.

El Marjal Pego-Oliva constituye la zona húmeda más septentrional de la Provincia de Alicante y se formó por la colmatación de una albufera, originada por los depósitos de los ríos que lo delimitan. La declaración de Parque Natural y la consiguiente protección por parte del Estado Español, la Unión Europea y la Comunidad Valenciana, no ha sido suficiente para impedir algunas actuaciones. En opinión de algunos la extensión de la zona protegida del parque ha pasado de 900 Ha en 1996 a 345 en el 2000 y la proporción del marjal del total del parque ha pasado del $70.3 \%$ al $26.9 \%$, en este mismo periodo (La Casa Verde, 2000).

Tradicionalmente los estudios que se realizan en nuestro país sobre este tipo de ambientes acuáticos se centran en el estudio de las aves o de algún otro grupo animal, quedando relegadas a un segundo término las plantas y las algas. Sin embargo, el Marjal de Pego-Oliva alberga una riqueza florística y unas formaciones vegetales que contienen todavía especies y asociaciones de alto valor corológico, por estar muy reducida su área de distribución o haber desaparecido del resto de las zonas húmedas del litoral mediterráneo (Boira, 1988). El gradiente de salinidad desde aguas dulces a salobres y los diferentes niveles de eutrofia que presenta este humedal, permiten también la presencia de una alta diversidad de macro y microalgas, que la convierten en una zona de gran interés en términos de biodi- versidad para la Península Ibérica y para el continente europeo. A pesar de ello son pocos los datos que tenemos en la actualidad sobre las comunidades vegetales de este marjal (Boira, 1988; Box 1987; Carretero 1990; Margalef-Mir 1981). Más fragmentaria es, todavía, la información relativa a los grupos algales (Aboal \& Fletcher, 1997; Carretero 1990; Comín, 1989; Comín \& Tomás 1985; Margalef-Mir 1981;
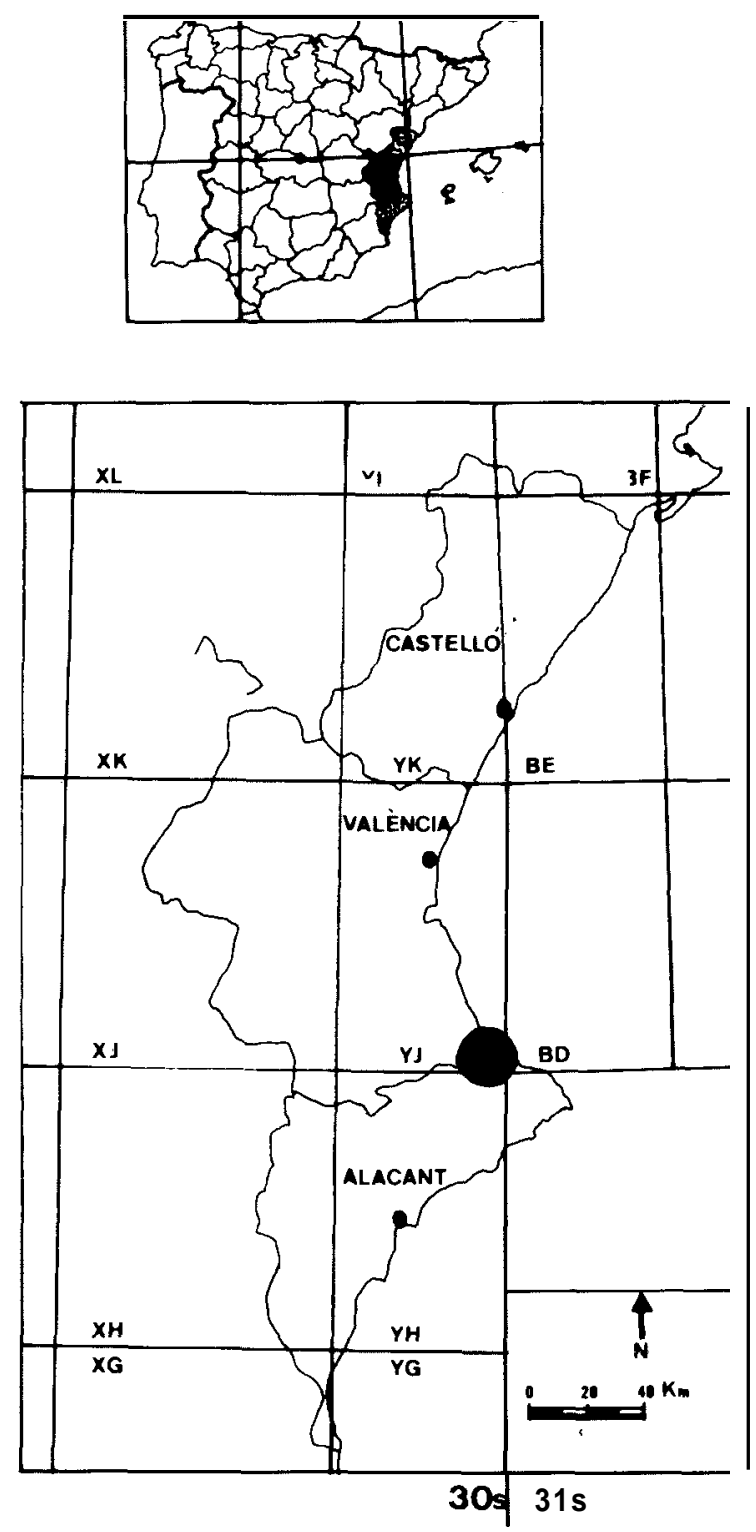

Figura I. Localización geográfica de la zona de estudio. Study area 
Tomás et al, 1980; Tomás, 1981, 1988). En ningún caso se ha realizado un estudio sistemático del área, sino que más bien se pone de manifiesto el interés de las especies recolectadas en visitas puntuales.

Con este trabajo queremos hacer una aproximación al estudio de la flora algal de este Marjal, particularmente de las macroalgas, así como a los cambios que se han producido en este sistema acuático en los últimos veinte años, para tratar de valorar su estado de conservación.

\section{AREA DE ESTUDIO}

Su superficie se reparte entre los términos municipales de Oliva (en la provincia de Valencia) y Pego, Denia y Vergel (en la provincia de Alicante). Presenta una forma alargada, paralela a la costa con unos $14 \mathrm{Km}^{2}$ de superficie (Fig. 1).El marjal se encuentra inmerso en un conjunto de elevaciones prebéticas. La dirección dominante del conjunto montañoso es SW/NE, siguiendo las direcciones generales del prebético. El límite septentrional es la Sierra de Mustalla (200 m), por el sur están las sierras de Castellá, Caral y Segaria (500-600 m), por el este el límite está constituido por un conjunto de sierras, de las que las de mayor cota son las de Almiserá y Tossal Gran (700 m). El límite por el este, y la separación del mar, es una restinga arenosa de $9 \mathrm{~km}$ de longitud y 1.6 $\mathrm{km}$ de anchura. En su formación han intervenido los depósitos de los ríos Gallinera, Bullent y Racons. Los procesos posteriores de colonización vegetal y colmatación han producido una elevación del terreno lacustre y la formación de terrenos turbosos (Box, 1987; Viñals et al., 1990).

El río Bullent recorre el marjal por su flanco septentrional y recoge los aportes de diversos manantiales de agua dulces y otros de agua salada. El río Racons recorre el extremo meridional del marjal y recoge las aguas de algunos barrancos y los aportes subterráneos de la Bassa Sineu, al pie de la sierra de Segaria. El río Revuelta recorre el marjal de norte a sur y sirve de comunicación entre los dos ríos anteriores. Al mismo tiempo existen numerosos canales, tanto de riego como de avenamiento, fruto del uso tradicional de los terrenos para la agricultura (Urios et al., 1993), así como dos estaciones de bombeo hacia los ríos Bullent-Vedat y Racons-Regalacho (Diputación Provincial de Alicante, 1992).

Los materiales geológicos más antiguos son calizas y dolomías, que conforman las vertientes de las montañas que rodean la cuenca, mientras que en el marjal predominan las margas y los sedimentos fluviales.

Las precipitaciones en esta zona suelen ser torrenciales con una media anual de $905 \mathrm{~mm}$ en Pego, con máximos otoñales y primaverales y una acusada sequía estival (Viñals et al., 1990; Varios autores, 1984). La temperatura media anual es de $18^{\circ} \mathrm{C}$, los veranos son cálidos con temperatura media de $25-26^{\circ} \mathrm{C}$ (en agosto) y los inviernos suaves con $10.9^{\circ} \mathrm{C}$ de media (en enero). El año 1999 ha resultado ser el más seco del siglo.

Se inscribe en el piso termomediterráneo ombroclima subhúmedo (Rivas Martínez, 1987). Por la humedad ambiental reinante en la época de verano esta definición podría ser matizada para incluirla, durante este periodo, en el tipo climático subtropical subhúmedo (Boira, 1988).

\section{MATERIAL Y MÉTODOS}

Las visitas iniciadas en el año 1995 se realizaron preferentemente en los momentos de máxima precipitación y mayor desarrollo de las comunidades vegetales (primavera) y/o en los momentos de mínima inundación (verano). Se han muestreado un total de 7 estaciones, desde la parte norte a la sur del Marjal (Fig. 1). Los ambientes muestreados en las diferentes épocas en la zona de estudio han sido corrientes dulces, corrientes salobres, nacimientos dulces y salobres, pozas salinas, charcas someras, azarbes y arrozales. Se han recolectado comunidades algales bentónicas, separadas del substrato con ayuda de espátulas o navajas. Las variables fisicas y químicas se han determinado in situ: $\mathrm{pH}$, temperatura, conductividad, oxígeno disuelto y velocidad de corriente. Se tomaron muestras de agua y 
se congelaron en el campo para evaluar los principales aniones y cationes por cromatografía iónica (servicio de análisis de agua del SACE, Universidad de Murcia). El material vegetal se transportó en frío hasta el laboratorio para su observación y posteriormente se fijó con formaldehido y/o se prensó. Todo el material está depositado en el Herbario de la Universidad de Murcia (MUB. ALGOLOGIA).

La observación microscópica se realizó descarbonatando previamente con EDTA. Las diatomeas se limpiaron con agua oxigenada en caliente y se montaron en Naphrax. Para la observación con Microscopio electrónico de barrido se metalizó el material previamente limpio con oro-paladio.

En algunos grupos se trató de inducir la formación de estructuras reproductoras en semicultivos mantenidos en las condiciones del laboratorio.

\section{RESULTADOS.}

\section{Periodo 1995-1999.}

La zona de estudio reúne una serie de ambientes acuáticos con diferentes calidades de agua (Tabla 1), desde arroyos de agua dulce (Bullent), hasta nacimientos de agua dulce (ullals), pasando por azarbes de riego, más o menos eutrofizados, surgencias termales de aguas salobres (Font Salat), surgencias de aguas salobres no termales (Bassa Sineu), ríos eutrofizados y contaminados (Racons).

El aspecto más relevante que se ha observado durante los años de estudio es la disminución de los caudales de todos los ambientes y una disminución de la transparencia de las aguas. Sin embargo, la composición química ha permanecido notablemente constante, como se puede apreciar al comparar la evolución de los puntos en los últimos cinco años (Tabla 1). También es manifiesta la disminución en la conductividad de las aguas de la Bassa Sineu y una modificación en las proporciones aniónicas y catiónicas. En general, existe suficiente cantidad de nutrientes para permitir un crecimiento vegetal importante, sobre

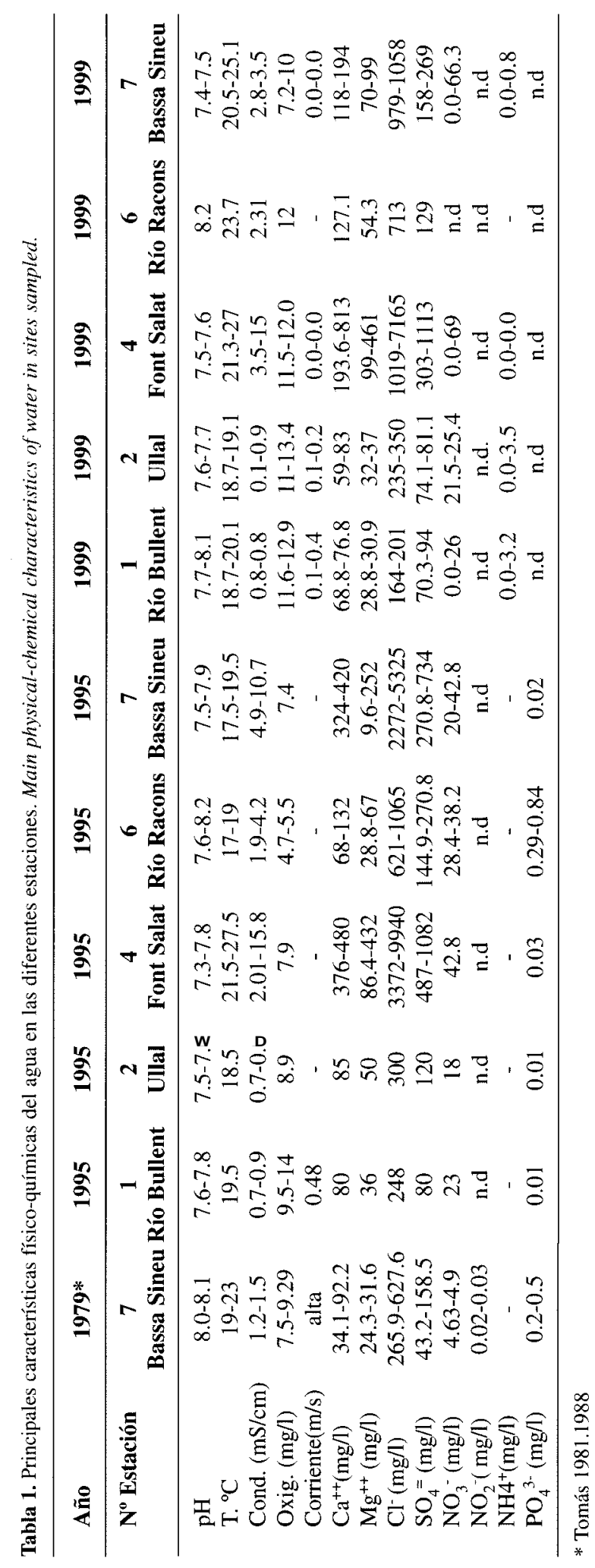


todo de nitratos ya que los fosfatos pueden ser indetectables gran parte del año, al quedar retenidos en los precipitados de carbonatos. El grado de eutrofización no parece haber variado con los años, a pesar de la disminución apreciada en los caudales. Dentro del sistema coexisten ambientes con diferente grado de trofía, que puede provocar un aumento en la riqueza específica. Un buen ejemplo de esto son los azarbes que pueden albergar innumerables especies de flagelados (nunca estudiados en profundidad), además de plantas vasculares flotantes como las lentejas de agua que pueden servir de soporte a especies muy interesantes y poco citadas en nuestro país, como Chaetotheke reptans, Chlorochytrium paradoxum o Gongrosira disciformis .

En la actualidad los sistemas dulciacuícolas están sobre todo extendidos en la parte norte, en el término municipal de Oliva. En las primeras visitas al marjal (en 1995), en el río Bullent se desarrollaban crecimientos importantes de Batrachospermum boryanum o de Draparnaldia glomerata sobre los macrófitos, o incluso algunas praderas de Chara hispida (Tabla 2). En la actualidad estas especies han desaparecido, al menos en el tramo visitado.

En las riberas del río Bullent existen diversos ullales, con diferentes grados de conservación. En los Últimos años también se ha podido comprobar la práctica desaparición de algunas especies, como Batrachospermum boryanum, debido a que artificialmente se han estancado sus aguas, impidiendo su natural discurrir hacia el río. Esta rodofícea necesita aguas bien oxigenadas y sólo se desarrolla en condiciones de corriente más o menos intensa. En los canales de desague de alguno de estos ullales, si existe circulación del agua y condiciones esciófilas se desarrolla Hildenbrandia angolensis.

Existe otro tipo de ullales situados en el término municipal de Pego, como la Bassa Sineu, caracterizados en la actualidad por sus aguas más mineralizadas u oligohalinas. En las primeras visitas realizadas (en 1995) se pudo comprobar el desarrollo profuso de Compsopogon coeruleus, y de unas ricas comunidades de diatomeas epífitas sobre Potamogeton pectinatus que era la única planta vascular presente. Polysiphonia subtilissima formaba penachos muy conspicuos sobre todo tipo de substratos. Las características generales de estos enclaves permanecen más o menos invariables, pero se ha producido un descenso en la salinidad del agua. En muchos casos también se ha aumentado artificialmente el tiempo de retención, al impedir el flujo de sus aguas. Esto ha conllevado un aumento de turbidez que impide el desarrollo de las comunidades de angiospermas y algas que allí habitaban. En la actualidad no se aprecian tampoco desarrollos macroalgales, sino solamente de microalgas como quetoforales, oscilatoriales y diatomeas. Sin embargo, algunos táxones como Compsopogon coeruleus permanecen en el sistema, no en forma macroscópica, sino en forma de células de resistencia, como se pudo comprobar en cultivos realizados a partir de raspados de superficies sumergidas.

El río Racons se caracteriza por su carácter oligohalino y por el elevado grado de eutrofia que presentan sus aguas, debido al impacto de las urbanizaciones que flanquean el marjal por el sur. En las primeras visitas (en 1995), en la época primaveral se podían contemplar enormes desarrollos de Compsopogon coeruleus, Cladophora sp. y Enteromorpha intestinalis, que posteriormente desaparecieron por completo. La puesta en marcha de una depuradora ha permitido mejorar la calidad de las aguas, hasta el punto que empiezan a observarse las especies que habían desaparecido.

La Font Salat es un manantial termal de aguas saladas, que es visitado por los vecinos del lugar por el carácter benéfico de sus aguas. El fondo limoso está ocupado por praderas de Potamogetonpectinatus, sobre el que se asientan un buen número de especies epífitas. Sobre las rocas del perímetro pueden observarse los penachos violáceos de Polysiphonia subtilissima (que también se desarrolla profusaniente como epífito), Chaetomorpha sp., Cladophora sp. Entre las diatomeas que pueden formar cadenas visibles en el campo, se pueden citar Terpsinoe musica, Hyalodiscus whitneyi y Melosira moniliformis (junto con la var. octagona). Rodeando este enclave, existen otras surgencias, algo menos salinas, en las que se desarrolla Thorea violacea, 
Tabla 2. Especies de macroalgas recolectadas en el Marjal de Pego-Oliva. Se han marcado con negrita los taxones que sólo se conocen del área o que tienen una distribución escasa en la Península Ibérica. Macroalgae spp. Collected from Marsh Pego-Oliva. In bold are taxu only known to the arm or generally scarce across the Iberian Peninsula.

\begin{tabular}{|c|c|c|c|c|}
\hline Especies/Colectas & $1979 *$ & $1990 * *$ & 1995 & 1999 \\
\hline \multicolumn{5}{|l|}{ CYANOPHYCEAE } \\
\hline Calothrix parietina Thur. & & & 2 & 2 \\
\hline Calothrix pulvinata $\mathrm{Kg}$. & & & & 4 \\
\hline Homoeothrix crustacea Woron. & & & 1 & 1 \\
\hline Homoeothrix juliana (Menegh.) Kirchn. & & & 1,2 & 1,2 \\
\hline Homoeothrix violacea (Born. et Flah.) Komárek \& Kann & & & & 4 \\
\hline Lyngbya martensiana Menegh. & & & & 4 \\
\hline Microcoleus sp. & & & & B \\
\hline Oscillatoria sp. & A & & & 1 \\
\hline Phormidium angustissimum W. et. G.S. West & & & 2 & 2 \\
\hline Phormidium sp. & & & & 2 \\
\hline Phorrnidium tenue (Menegh.) Gom. & & & & 4 \\
\hline Schizothrixfasciculata (Kütz.) Gom. f. semiglobosa & & & 1,2 & 1,2 \\
\hline \multicolumn{5}{|l|}{ RHODOPHYCEAE } \\
\hline Batrachospermum boryanum Sirodot. & & & 1,2 & 2 \\
\hline Compsopogon coeruleus (Balb.) Mont. & A & & $3,5,6,7$ & $3,5,7$ \\
\hline Hildenbrandia angolensis Welwitsch ex W. West \& G.S. West & & & & 2 \\
\hline Polysiphonia subtilissima Montagne & & & $3,4,6,7$ & $3,4,6,7$ \\
\hline Thorea ramosissima Bory De Saint-Vincent & A & & & \\
\hline Thorea violacea Bory de Saint-Vincent & & & & 4 \\
\hline \multicolumn{5}{|l|}{ CHLOROPHYCEAE } \\
\hline Chaetophora elegans Ag. & & & 1 & 1 \\
\hline Chaetophora cf. pisiformis (Roth) Ag. & & & & 1,2 \\
\hline Chaetophorales indeterminadas & & & & 7 \\
\hline Chaetotheke reptans Düringer & & & 5 & \\
\hline Chlorochytrium paradoxum (Klebs) G.S. West & & & 5 & \\
\hline Draparnaldia glomerata Ag. & & & 1 & \\
\hline Gongrosira disciformis & & & 5 & \\
\hline Hydrodyction reticulatum (L.) Lagerh. & & & 5 & \\
\hline Oedogonium sp. & 4 & & & 5 \\
\hline Sphaeroplea annulina (Roth) Ag. & & & & $\mathrm{C}$ \\
\hline \multicolumn{5}{|l|}{ ULVOPHYCEAE } \\
\hline Cladophora fracta (Müller ex Vahl) Kütz. & $\mathbf{A}$ & & 4,7 & 4,7 \\
\hline Cladophora glomerata (L.) Kütz. & & B & I & 1 \\
\hline \multicolumn{5}{|l|}{ Cladophora glornerata var. crassior } \\
\hline Chaetomorpha linum & & B & 4 & 4 \\
\hline Enteromorpha clathrata (Roth) Grev. & & $\mathrm{B}$ & & \\
\hline Enteromorpha flexuosa (Wulfen ex Roth) Ag. & A & $\mathrm{B}$ & & \\
\hline Enterornorpha intestinalis (L.) Link & & $\mathrm{B}$ & 4,7 & 4,7 \\
\hline $\begin{array}{l}\text { Rhizoclonium hieroglyphicum (Kütz.) Stock. } \\
\text { CHAROPHYCEAE }\end{array}$ & & $\mathrm{B}$ & 4 & 4 \\
\hline Coleochaete scutata Bréb. & & & 5 & 5 \\
\hline Chara aspera Deth. ex Willd. & & $\mathrm{B}$ & & \\
\hline Chara braunii Gm. & & & & $\mathrm{C}$ \\
\hline Chara globularis Thuill. & & $\mathrm{B}$ & & \\
\hline Chara hispida $\mathrm{L}$. & & $\mathrm{B}$ & 1 & \\
\hline Chara vulgaris $\mathbf{L}$. & & $\mathrm{B}$ & $\mathrm{B}$ & $1, \mathrm{C}$ \\
\hline Chara vulgaris var. contraria Braun ex Kütz. & & & & $\mathrm{B}$ \\
\hline Mougeotia sp. & $\mathbf{A}$ & & & 5 \\
\hline
\end{tabular}




\begin{tabular}{|c|c|c|c|c|}
\hline Nitella batrachosperma(Reich.) A. Braun & & & 5 & 5 \\
\hline Nitella hyalina $(D C) A g$. & & & 5 & 5 \\
\hline Spirogyra affinis (Hassall) Petit & & $\mathrm{B}$ & & \\
\hline Spirogyra condensata (Vauch.) Kütz. & & $\mathrm{B}$ & & \\
\hline Spirogyra majuscula Kütz. & & $\mathrm{B}$ & & \\
\hline Spirogyra semiornata Jao & & $\mathrm{B}$ & & \\
\hline Spirogyra sp. & A & & & 5 \\
\hline Spirogyra varians (Hassall) Kütz. & & $\mathrm{B}$ & & \\
\hline Tolypella glomerata (Desv. in Lois.) Leon. & & & & $\mathrm{B}$ \\
\hline Zygnema stellinum (Vauch.) Ag. & & $\mathrm{B}$ & & 5 \\
\hline Zygnema sp. & A & & & \\
\hline \multicolumn{5}{|l|}{ XАNTHOPHY CEAE } \\
\hline Vaucheria dichotoma (L.) Ag. & & $\mathrm{B}$ & $\mathrm{B}$ & $\mathrm{B}$ \\
\hline Vaucheria sp. & & & & 1,2 \\
\hline \multicolumn{5}{|l|}{ BACILLARIOPHY CEAE } \\
\hline Hyalodiscus whitneyi Ehr. & 7 & & 4 & 4 \\
\hline Melosira moniliformis (O.F. Müller)Ag. & 7 & & 4 & 4 \\
\hline Melosira moniliformis var. octagona (Grun.)Hust. & 7 & & 4 & 4 \\
\hline Terpsinoe musica Ehr. & 7 & & 4 & 4 \\
\hline
\end{tabular}

* Tomás 1981, 1988

${ }^{* *}$ Carretero 1990

acompañada por Polysiphonia subtilissima, Enteromorpha intestinalis y Cladophora sp.

Los azarbes pueden albergar comunidades complejas de hidrófitos junto con algas como Hydrodyction reticulatum o rodofíceas como Compsopogon coeruleus. Las praderas de Nitella batrachosperma pueden ser importantes puntualmente en primavera. Sin embargo muchos de estos azarbes en la actualidad sólo están ocupados por Cladophora.

Las charcas que se forman tras las lluvias se cubren con rapidez de caráceas como Chara vulgaris, Ch. vulgaris var. contraria o Tolypella glomerata, junto con Vaucheriadichotoma.

Finalmente los arrozales albergan un gran número de especies de micro y macroalgas, de entre ellas cabe señalar por su rareza en un contexto nacional: Chara braunii y Sphaeroplea annulina.

En la figura 2 se puede observar la susbstitución de especies de macroalgas producida en los últimos cinco años en el marjal (desde 1995-1999).
Durante los años de este estudio se han reconocido un total de 58 taxones de macroalgas y de ellas el 50\% (aprox.) son nuevas citas para el área (Tabla 2). La misma proporción de novedades se produce con las diatomeas (Cantoral, en prensa). Algunas de las especies tienen aquí su única cita conocida en toda España y en todo el continente europeo: Thorea violacea, y otras tienen una distribución restringida: Compsopogon coeruleus, Hildenbrandia angolensis, Polysiphonia subtilissima, Sphaeroplea annulina, Chara braunii, Nitella batrachosperma, Nitella hyalina, Hyalodiscus whitneyi, Melosira moniliformis, Melosira monilijormis var. octagona y Terpsinoe musica (Tabla 2).

\section{Datos previos}

De los primeros puntos ( 1 y 2 ) carecemos de datos previos de algas o de comunidades vegetales. Sólo Carretero (1990) menciona para el punto tres a Enteromorpha intestinalis. 

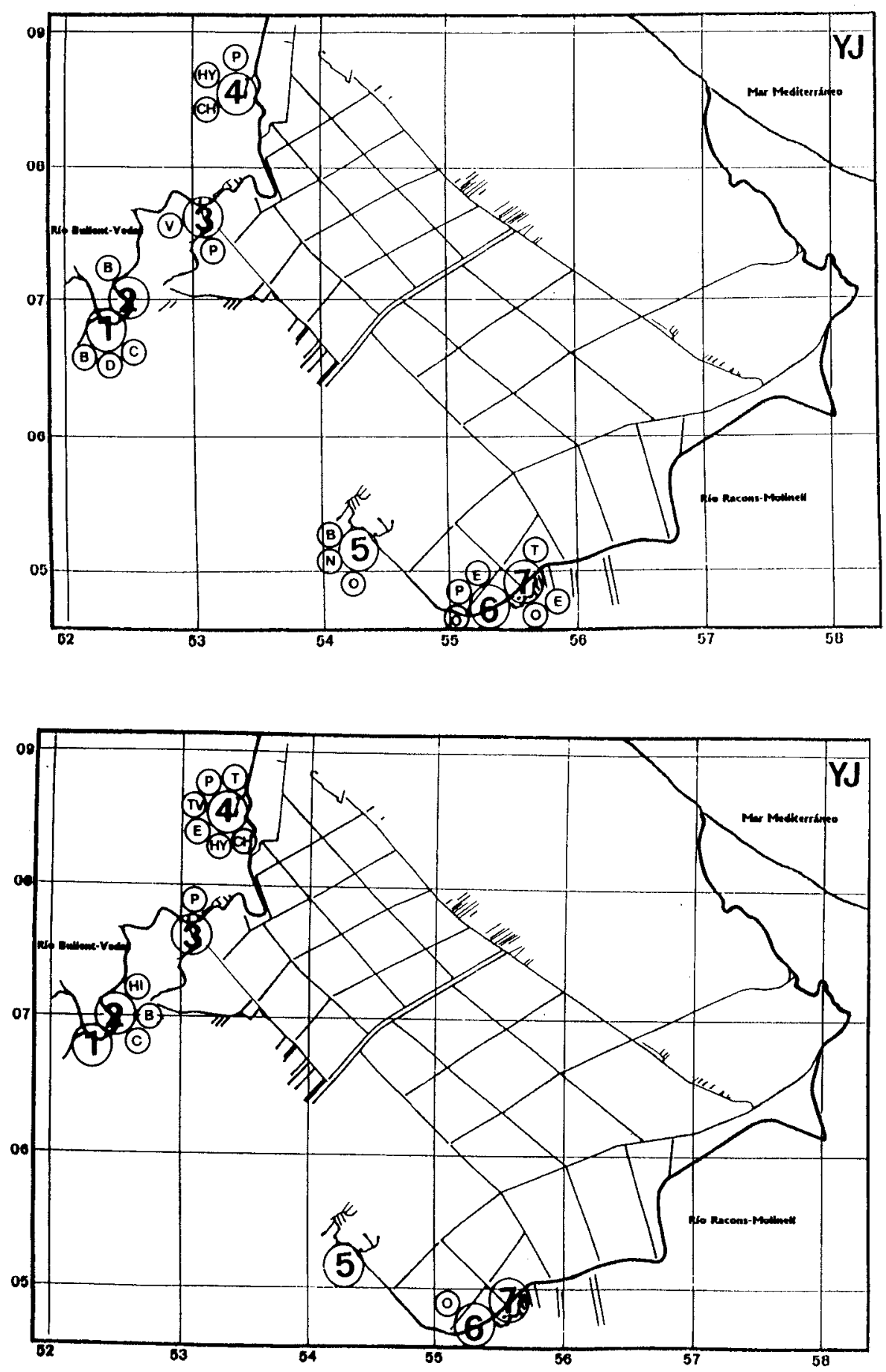

Figura 2. Variación de las especies de macroalgas entre a) 1995 y b)1999. Variablity of the macroalgae species between a) 1995 and b) 1999. $(\mathbf{P}=$ Polysiphonia sultilissima, $\mathbf{H Y}=$ Hyalodiscus whitneyi, $\mathbf{C H}=$ Chaetomorpha $\mathbf{s p} ., \mathbf{C}=$ Chaetophora elegans, $\mathbf{B}=$ Batrachospermum boya . num,, $\mathbf{V}=$ Vaucheria $\mathbf{s p} ., \mathbf{E}=$ Enteromorpha $\mathbf{~} \sim, \mathbf{O}=$ Compsopogon coeruleus, $\mathbf{N}=$ Nitella batrachosperma, $\mathbf{T}=$ Terpsinoe musica, $\mathbf{T V}=$ Thorea violacea, $\mathbf{H I}=$ Hildenbrandia angolensis).. 
Los datos de 1979 de los ullales de la zona de la Bassa Sineu, se refieren a lo que Tomás (1987) llama manantiales de Pego, que presentaban una mineralización baja $(<1500 \mu \mathrm{S} / \mathrm{cm})$, con predominancia de los cloruros frente a los sulfatos debido a la influencia del agua freática, con alcalinidad $(<6$ meq/l) debida fundamentalmente al $\mathrm{HCO}_{3}{ }^{-}$que es el anión más abundante, y con el $\mathrm{Ca}^{++}$y el $\mathrm{Na}^{+}$ que se alternan en el dominio catiónico con concentraciones de nitratos y fósforo moderadas. $\mathrm{La}$ mineralización asegura que es la principal causante de las diferencias en la composición de las poblaciones de diatomeas. En algunas publicaciones emanadas de esta misma expedición (MargalefMir, 1981,Tomás 1981, Tomás et al., 1980,Comín 1989, Comín \& Tomás, 1985) se añaden algunas descripciones adicionales de los puntos en los que se recolectaron macroalgas como Thorea hispida $(=$ T. ramossissima) y Compsopogon coeruleus. Paralelamente se comenta la gran transparencia de las aguas y la diversidad específica de las comunidades de plantas vasculares.

En el río Racons, Carretero (1990) menciona a Cladophora glomerata, Enteromorpha clathrata y E. fexuosa.

Quizá el tipo de ambiente más intensamente estudiado y del que se posee un mayor número de datos es de los canales de riego y azarbes. En estos sistemas Carretero (1990) señaló Chara hispida, Cladophora glomerata, Enteromorpha flexuosa, Enteromorpha intestinalis y Spirogyra varians.

Las charcas y pozas más o menos someras que se producen tras las lluvias son colonizadas por: Chara aspera, Ch. globularis, Ch. hispida, Ch. vulgaris, Cladophora glomerata, Spirogyra affinis, S. condensata, S. semiornata y Vaucheria dichotoma (Carretero 1990).

En el periodo comprendido entre 1979 y 1995 (Tabla 2) se habían reconocido un total de 29 especies de macroalgas (que desarrollan crecimientos conspicuos a simple vista).

\section{DISCUSION}

En total, en los estudios que se han realizado en el Marjal de Pego-Oliva hasta la fecha, se han reconocido 60 taxones (Cyanophyceae 12, Rhodophyceae 6, Chlorophyceae 9, Ulvophyceae 8, Charophyceae 19, Xanthophyceae 2 y Bacillariophyceae 4 (Tabla 2).

En la figura 3 se puede apreciar el incremento apreciado en la riqueza específica en este enclave en los últimos veinte años. A pesar de que no se ha realizado un estudio sistemático de la zona, más que en años recientes, se han observado una serie de cambios en la flora del Marjal Pego-Oliva.

Los efectos de la sequía, probablemente agravados por el aumento del agua bombeada, han provocado un descenso del nivel de agua muy acusado en todas las estaciones de muestreo visitadas. Asociado al descenso del nivel de las aguas se ha producido una disminución de la velocidad de corriente y un incremento de la turbidez, particularmente evidente en el río Bullent en verano, lo que ha inducido el desplazamiento o la desaparición de algunas especies, como Batrachos-

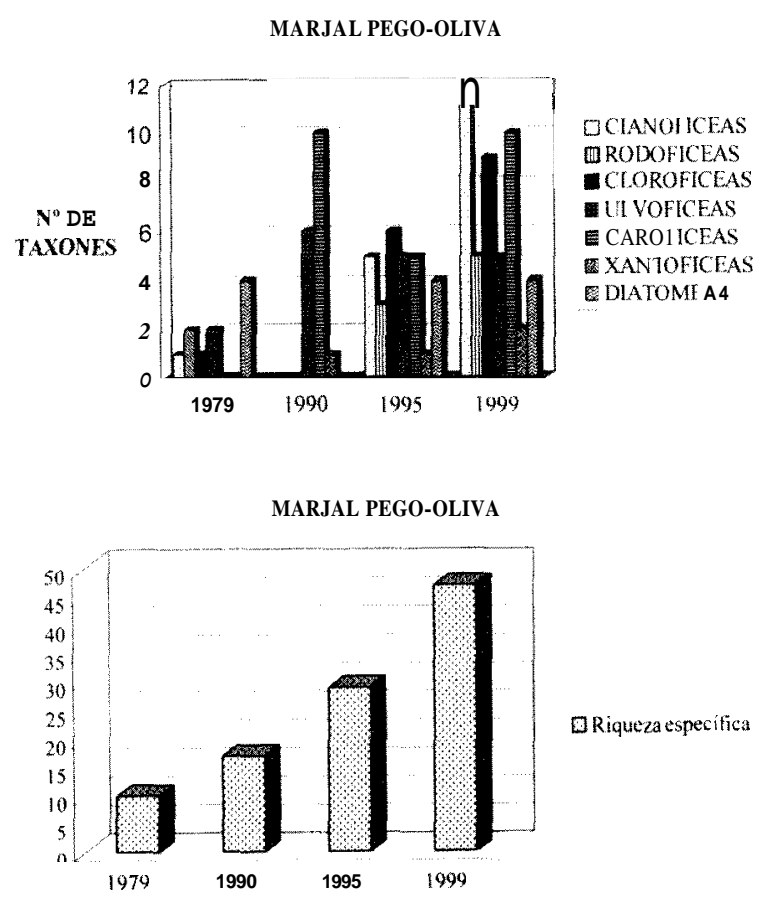

Figura 3. Evolución de la riqueza específica total y de las macroalgas de 1979-1999. Changes ojthe total specific ricchess and of the macroalgae between 1979 and 1999. 
permum boryanum y Draparnaldia glomerata que se han refugiado en algunos de los ullales que vierten al río Bullent o han desaparecido por completo. La modificación en las condiciones de estos ullales también hace peligrar su permanencia en ellos. Chaetophora elegans que es menos exigente puede encontrarse todavía aunque con menos frecuencia en el río, también en las proximidades de los ullales.

La disminución de los caudales tiene como consecuencia un aumento leve de la mineralización, patente en el río Bullent y en algunos de los ullales de la zona norte. En ambos casos se ha observado un empobrecimiento de las comunidades. La ubicación de los ullales entre los cultivos o próximos a las casas los hacen muy susceptibles a constantes manipulaciones, que suelen reducir el número de microambientes (en muchos casos se impide el normal discurrir de las aguas) y consiguientemente la diversidad. Pese a todo, se ha recolectado recientemente Hildenbrandia angolensis Welwitsch ex W. West $\&$ G.S. West, en un canal que vierte las aguas de un ullal al río Bullent, en zonas muy esciófilas. Esta rodofícea, descrita en Africa, tiene preferencia por las aguas cálidas, está extendida por el sur de los Estados Unidos (Sheath et al., 1993 c) y se ha citado recientemente para nuestro país (Ros et al., 1997).

Los bombeos abusivos que tratan de desecar terrenos pueden aumentar la intrusión del agua marina, debido a la debilidad de los acuíferos sobreexplotados. Además en algunos casos el manto freático se encuentra por debajo del nivel del mar (Bassa Sineu, por ejemplo), aumentando todavía más el riesgo de intrusión (Box 1987). Este hecho podría explicar la extensión en dirección norte de Polysiphonia subtilissima Montagne, una rodofícea de origen marino, que suele vivir en zonas costeras cálidas en donde se producen mezclas de agua marina y dulce. Esta especie de un porte considerable (hasta 12-15 $\mathrm{cm})$ no fue observada en los primeros estudios realizados en la zona, sin embargo hoy es abundante. La ausencia de procesos reproductivos es una característica de la especie, que suele desarrollarse en zonas de desembocaduras de arroyos en zonas cálidas (Taylor, 1960). Sheath \& Cole (1990) la citan en arroyos alimentados por manantiales en Florida, en donde convive con Terpsinoe musica y Compsopogon coeruleus, en condiciones ambientales muy parecidas a las del marjal. Algunos autores piensan que forma parte de un complejo de especies típicamente marinas que incluye a Polysiphonia urceolata, que se ha modificado morfológicamente y ha perdido la capacidad reproductora al adaptarse a vivir en aguas de baja salinidad (Maggs \& Hommersand, 1993). El papel de los freáticos es fundamental para explicar, por ejemplo, los cambios de salinidad experimentados en la Bassa Sineu (Tabla 1) y sus consiguientes cambios florísticos.

Los problemas de contaminación del río Racons, en los Últimos años han contribuido a la virtual desaparición de Compsopogon coeruleus. Se trata de una especie con claras afinidades tropicales que es muy rara en el continente europeo (Bourrelly, 1985), aunque solía estar bastante extendida por el litoral español (Tomás et al, 1980). Su rango ecológico ha resultado ser mucho más amplio del que inicialmente se pensaba, ya que se puede desarrollar tanto en aguas circulantes, dulces y muy puras, eutrofizadas o incluso contaminadas en países tropicales (Necchi \& Ribero, 1992; Carmona, 1998?), o en aguas salobres y con una carga orgánica importante como en este caso. En las primaveras solía formar grandes extensiones en la zonas encharcadas del carrizal, en donde podían observarse los talos viejos huecos flotando en la superficie del agua (aunque en las primeras fases del desarrollo se encuentren fijados a los culmos de la gramínea).

El mantenimiento de las áreas de arrozal también sirve para preservar la diversidad algal, como se ha dicho en innumerables ocasiones. Allí se pueden recolectar especies de caráceas que están muy escasamente citadas en nuestro territorio como Chara braunii o filamentosas como Sphaeroplea annulina.

Algunas especies características de aguas cálidas, como Thorea hispida $(=T$. ramossissima), que precisan para su desarrollo aguas muy puras no han sido reencontradas después de su primera cita en los ullales de Pego (Tomás, 1981). Esta 
especie se había citado también en el río Mosa en Bélgica (Descy \& Empain, 1974), en el Sena en París cuando la calidad del agua era mayor (Bourrelly, 1985) y en Alemania en zonas de aguas cálidas (Rott et al., 1999). En la actualidad parece que ha sido substituida por Thoreu violacea que prefiere aguas de mayor mineralización, aunque siempre también está relegada a la presencia de manantiales (Sheath et al., 1993b, Carmona, 1998). En esta zona se desarrolla en surgencias de aguas salobres (oligohalinas/mesohalinas) de corriente lenta, en un área muy reducida y es muy rara.

Las recientes obras de acondicionamiento realizadas en la Font Salat, con el objeto de facilitar el baño y las visitas turísticas, han modificadoel substrato original, arenoso-limoso, mediante la colocación de cantos, lo que indudablemente provocará modificaciones importantes a nivel florístico.

La intrincada red de azarbes distribuidos por el territorio ofrece toda una gama de características ambientales y muchos de ellos sirven de refugio de especies de plantas vasculares que tienen una distribución muy restringida a escala nacional, aunque pueden estar presentes en otros humedales de la Comunidad Valenciana (Cirujano, 1995).

Algunas especies de diatomeas que son características de climas cálidos, parecen haber aumentado en su abundancia en los Últimos años, algunas de ellas aparecían aunque eran muy raras como Hyalodiscus whitneyi y Terpsinoe musica. Se ha observado también un aumento en la abundancia de especies consideradas como poco frecuentes en trabajos anteriores (Tomás, 1988), que podrían avalar la tendencia al incremento local de la salinidad: Nitzschia gracilis Hantzsch, Rhoicosphenia abbreviata (C. Agardh) Lange-Bertalot, Cocconeis placentula var. euglypta (Ehrenberg) Grunow, Cocconeis placentula var. lineata (Ehrenberg) Van Heurck, Diploneis interrupta (Kützing) Cleve (Cantora1 $\&$ Aboal, en prensa).

A pesar de tratarse de un área que el hombre ha manejado desde antiguo, representa un refugio de diversidad, que es bastante excepcional entre los sistemas acuáticos del Levante peninsular, de la Península e incluso del continente euro- peo, ya que algunas de las especies sólo están citadas aquí para todo el territorio nacional: Terpsinoe musica (aunque existe una cita de principios de siglo de la costa murciana), Hyalodiscus whitneyi, Thorea hispida (probablemente desaparecida), Thorea violacea, Polysiphonia subtilissima, Chaetotheke reptans, Chlorochytrium paradoxum. Otras especies tienen una representación muy escasa en otras áreas: Hildenbrandia angolensis, Chara braunii, Sphaeroplea annulina.

El número total de especies reconocido en la actualidad probablemente está muy por debajo del real, entre otras cosas, por el enorme número de ambientes diferentes existente en la zona, muchos de ellos temporales o de difícil acceso, que hacen su muestreo bastante complicado. Hay que recordar que prácticamente todos los datos existentes y aportados en este trabajo se limitan a las macroalgas. El estudio de las microalgas no sólo incrementará notablemente la diversidad sino que además dará la medida exacta de la singularidad de la zona.

Incluso las plantas vasculares han sufrido una regresión en los últimos años, para la que ya se han propuesto medidas correctoras (Boira, 1988). Sin embargo la misma complejidad del sistema es su mejor salvaguarda, al coexistir ambientes con diferente calidad de agua y distintos niveles de perturbaciones. La existencia de un número tan grande de surgencias, de condiciones estables, hacen que las condiciones físicas y químicas se puedan recuperar con mayor facilidad, a pesar del gran número de impactos sufridos en los últimos años.

Aunque no existe ningún estudio limnológico exhaustivo del área, la información que aportan las plantas resulta de un enorme interés para poder interpretar la dinámica de este tipo de sistemas y su evolución histórica y evidencia la importancia de tomarlas en consideración en los planes de control de la calidad, del uso y gestión del agua. Para esto es necesario realizar un seguimiento de estos grupos tan sensibles a los cambios ambientales de forma regular, realizando muestreos sistemáticos, al menos, en los momentos de máximo y mínima inundación. 


\section{AGRADECIMIENTOS}

Nuestro agradecimiento al apoyo otorgado a E. Cantora1 como Beca de Postdoctorado por la Dirección General de Asuntos del Personal Académico (DGAPA) de la Universidad Nacional Autónoma de México (UNAM) y al Departamento de Biología Vegetal (Botánica) de la Universidad de Murcia, España por la infraestructura y las facilidades prestadas para llevar a cabo la estancia de investigación. Luís Fletcher colaboró en algunas de las salidas al campo y con algunos análisis químicos.

\section{BIBLIOGRAFÍA}

ABOAL, M. \& L. FLETCHER. 1997. Clorofíceas asociadas a lentejas de agua: Chaetotheke reptans Düringer (Chaetosphaeridiaceae) Y Chlorochytrium paradoxum (Klebs) G. S. West (Chlorochytriaceae) primeras citas para la Península Ibérica y nuevos datos sobre Gongrosira disciformis Fritsch (Chaetophoraceae). Anales Jard. Bot. Madrid, 55(2): 457-458.

ADAM, P. 1990. Saltmarsh Ecology. Press Syndicate of Universitu of Cambridge. Cambridge.

BOIRA, H. 1988. Flora y Vegetación del Marjal de Pego-Oliva. Ayuntamiento de Oliva. $191 \mathrm{pp}$.

BOX, M. 1987 Los espacios anfibios litorales: albuferas y marjales: 129-152 En: Humedales y Úreas lacustres de la provincia de Alicante. Inst. Est. Juan Gil-Albert. Diputación Provincial de Alicante.

CANTORAL, E. \& M. ABOAL. (en prensa) Annual dynamics of diatom communities from Marjal Pego-Oliva, Alicante, Eastern Spain. Archiv. Hydrobiol. (Algological Studies).

CARMONA, J. 1998. Estudio florístico (taxonómicoecológico-biogeogrújico) de las rodófitas de agua dulce en la región central de México. Tesis Doctoral, Universidad Nacional Autónoma de México. $201 \mathrm{pp}$.

CARRETERO, C.J.L. 1990. Macrófitos acuáticos de la provincia de Alicante. Medi Natural , 2: 45-55.

CIRUJANO, S. 1995. Estudio de la flora y vegetación de las marjales de Pego-Oliva y la Safor, orientado a sugestión. Informe de la Consellería de Medio Ambiente de Valencia.

COMÍN, F. 1989. Els sistemes aquatics costaners. En: Historia Natural dels Països Catalans. 14.
Sistemes Naturals. Terradas, J., N, Prat, A. Escarré, i R. Margalef. (eds). Enciclopedia Catalana S.A. Barcelona.

COMÍN, F. \& X. TOMÁS. 1985. Els ambientes continentals rics en algues. En: Historia Natural dels Països Catalans. 14. Sistemes Naturals. Terradas, J., N, Prat, A. Escarré, i R. Margalef. (eds). Enciclopedia Catalana S.A. Barcelona.

DESCY, J.P. \& A. EMPAIN. 1974. Thorea ramossissima Bory (Rhodophyceae, Nemalionales) dans le basin Mosan belge. Bull. Soc. Roy. Bot. Belg., 107: 23-26.

DIPUTACIÓN PROVINCIAL DE ALICANTE. 1992. Mapa del Agua. Provincia de Alicante.

LA CASA VERDE. Butlletí nº5. 2000.

MATAECHE, P. 1998. Catálogo de las zonas húmedas de la Comunidad Valenciana (España).SEHUMED.

MARGALEF-MIR, R. 1981. Distribución de los macrófitos de las aguas dulces y salobres del E y NE de España y dependencia de la composición química del medio. Fundación Juan March. Serie Universitaria. $157 \mathrm{pp}$.

MAGGS, C.A. \& M.H. HOMMERSAND. 1993. Seaweeds of the British Isles. V.1. Rhodophyta. Part 3 A Ceramiales. HMSO. London.

MITSCH, W. \& GOSSELINK, J. 1993. Wetlands.Van Nostrand Reinhold.

NECCHI, O. \& M. RIBERO. 1992. The family Compsopogonaceae (Rhodophyta) in Brazil. Algological Studies, 66: 105-118.

RIVAS MARTÍNEZ, S. 1987. Memoria del mapa de vegetación de España. ICONA. Madrid.

ROS, M.D., E. LÓPEZ-JIMÉNEZ \& M. ABOAL. 1997. Primera cita de Hildenbrandia angolensis Welwitsch ex W. West \& G. S. West (Hildenbrandiales, Rhodophyceae), para la flora algal epicontinental española. Anal. Jard. Bot. Madrid, 55:458-460.

ROTT, E., E. PIPP, P. PFISTER, H. VAN DAM, K. ORTLER, N. BINDERN \& K. PALL. 1999. Indikationlisten für Aufwuchsalgen in Österreichischen Fliessengewüssern, Teil 2: Trophieindikation sowie geochemische Prüferenz, taxonomischen und toxicologische Anmerkungen. WWK, Bundesministerium für Land- und Forstwirtschaft. Wien.

SHEATH, R.G. \& J.M.BURKHOLDER. 1983. Morphometry of Batrachospermum population, intermediate between $B$. boryanum and B. ectocarpum (Rhodophyta). J. Phycol., 19: 324-331.

SHEATH, R.G. \& K.M. COLE. 1990. Batrachospermum heterocosticum sp. nov. and Polysiphonia 
subtilissima (Rhodophyta) from Florida spring-fed streams. J. Phycol., 26: 563-568.

SHEATH, R.G., D. KACZMARCZYK \& K.M. COLE. 1993. Distribution and systematics of freshwater Hildenbrandia (Rhodophyta, Hildenbrandiales ) in North America. Eur. J. Phycol., 28: 115-121.

SHEATH, R.G., M.L. VIS \& K.M. COLE. $1993 \mathrm{a}$. Distribution and systematics of Batrackospermum (Batrachospermales, Rhodophyta) in North America. Section Setacea. J. Phycol., 29: 719-725. SHEATH, R.G., M.L. VIS \& K.M. COLE. 1993 b. Distribution and systematics of the freshwater red algal family Thoreaceae in North America. Eur. $J$. Phycol. 28: 231-241.

TAYLOR, W.R. 1960. Marine Algae of the Eastern Tropical and Subtropical Coasts of America. University of Michigan Press, Ann Arbor.

TOMÁS, X., P. LOPEZ, R. MARGALEF-MIR \& F. COMIN. 1980. Distribution and ecology of Compso- pogon coeruleus (Balbis)Montagne (Rhodophyta, Bangiophycideae) in Eastern Spain. Cryptogamie: Algologie, 1: 179-186.

TOMAS, X. 1981. Tkorea ramosissima en un canal del litoral Valenciano. Fol. Bot. Misc., 2: 71-74.

TOMAS, X. 1988. Diatomeas de las aguas epicontinentales saladas del litoral mediterráneo de la península Ibérica. Tesis Doctoral, Universidad de Barcelona.

URIOS, V., P. DONAT \& M.J. VIÑALS. 1993. La Marjal de Pego-Oliva. El Medi Natural de la Marjal de Pego-Oliva i el seu entorn. Institut d'Estudis comarcals de la Marina Alta. Pedreguer.

VIÑALS, M.J., A.M. CAMARASA \& A. SENDRA. 1990. Factores de estabilidad en un humedal costero: la Marjal de Oliva-Pego. En: Actas 14 Reunión Nacional de Geomorfología. Tomo I. M. Gutiérrez, J.L. Peña y M.V. Lozano (Eds.):385-396 Instituto de estudios Turolenses. 